\title{
Is the middle cerebral artery bifurcation aneurysm affected by morphological parameters of bifurcation?
}

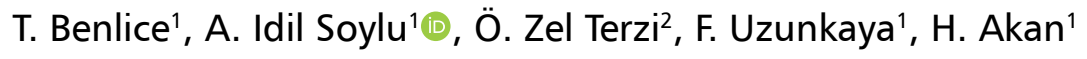 \\ ${ }^{1}$ Department of Radiology, OndokuzMayis University, Faculty of Medicine, Samsun, Turkey \\ ${ }^{2}$ Department of Public Health, OndokuzMayis University, Faculty of Medicine, Samsun, Turkey \\ [Received: 10 June 2020; Accepted: 25 June 2020; Early publication date: 14 August 2020]
}

Background: Aneurysm formation is a multifactorial process involving genetic, anatomical and environmental risk factors. A research focusing on the relationship between the presence of aneurysm and the morphology of the arteries will help in the pathogenesis and prediction of intracranial aneurysms. In this study, the relationship between the presence of aneurysm and various morphological parameters of aneurysm-related arteries was evaluated in patients with saccular middle cerebral artery (MCA) bifurcation aneurysm.

Materials and methods: The archival images of 74 patients (62.2\% women) were evaluated retrospectively. In this study, the angle between the ipsilateral MCA $M 1$ segment and the dominant truncus (Ф1), the angle between the $M 1$ segment and the recessive truncus (Ф2), and the bifurcation angle $(\Phi 1+\Phi 2)$ were compared. Bilateral internal carotid artery (ICA), MCA M1 segment, dominant and recessive truncus diameters and these diameters ratios were compared with the aneurysmal side and the contralateral side without aneurysm.

Results: When the dominant truncus, recessive truncus angles and bifurcation angle were compared, a significant difference was found on the aneurysmal side ( $p<0.0001)$. In the receiver operating characteristic analysis, when the bifurcation angle of $147.5^{\circ}$ was accepted as the limit value, $78.4 \%$ sensitivity, $79.7 \%$ specificity, $79.5 \%$ positive predictive value and $78.7 \%$ negative predictive value were determined (area under the curve: 0.85).

Conclusions: Our study of the morphological features of arteries associated with MCA bifurcation aneurysms showed that the presence of MCA aneurysms was significantly associated with large bifurcation angles. (Folia Morphol 2021; 80, 3: 520-526)

Key words: intracranial aneurysm, bifurcation morphology, haemodynamic changes

\section{INTRODUCTION}

Saccular intracranial aneurysms (IA) are potentially life-threatening vascular lesions. Due to the increase in the use of imaging techniques, 3-6\% of the aneurysms can be detected during the non-ruptured stage [22]. Bifurcation of the middle cerebral artery (MCA), which is one of the most common localisations of IA, accounts for approximately $20 \%$ of all IAs [6]. Although many studies have been conducted on the 
pathogenesis and localisation of IAs, the factors associated with aneurysm are still poorly understood.

Aneurysm formation is a multifactorial process involving genetic, anatomical and environmental risk factors. Familial inheritance and environmental factors such as smoking, alcohol use, hyperlipidaemia and hypertension increase the risk of IA development [9, 17, 21]. In addition to genetic and environmental factors, arterial morphology is thought to play an important role in aneurysm formation. Haemodynamic stress in the arterial bifurcation region can trigger aneurysm formation by triggering focal degenerative mechanisms in the vessel wall. Therefore, a research focusing on the relationship between the presence of aneurysm and the morphology of the arteries will help in the pathogenesis and prediction of IAs [23].

In this study, the relationship between the morphological parameters of the arteries around the aneurysm and the presence of aneurysm in patients with MCA bifurcation aneurysm was evaluated by comparing with the normal contralateral side.

\section{MATERIALS AND METHODS}

This study was approved by the institutional ethics committee. The requirement of informed consent was waived, as this was a retrospective study.

\section{Study population}

Between July 2015 and December 2018, patients who underwent brain computed tomography angiography (CTA) for cerebral aneurysm or subarachnoid haemorrhage in our hospital were retrospectively analysed. One hundred and nine patients with MCA aneurysm were selected for the study. Thirty five patients were excluded from the study: 10 patients with artefact images, 1 patient with severe atherosclerotic stenosis in internal carotid artery (ICA), 6 patients with bilateral aneurysm, 4 patients with trifurcation in MCA, 3 patients with severe vasospasm due to subarachnoid haemorrhage, 2 patients with M3 segment aneurysm and 9 patients with M1 segment aneurysm. Finally, 74 patients were included in the study. In order to minimise the effect of genetic and environmental risk factors on the development of aneurysm, the aneurysmal side and the contralateral side without aneurysm were compared in the same patient. We excluded patients with trifurcation due to the very low number of patients.

\section{CTA examinations}

Multidetector computed tomography (MDCT) shots were performed with Light Speed 64 General Electric Discovery CT750HD 2015 (Milwaukee, Wisconsin, USA). After 60-100 mL Optiray (Dublin, Ireland), a non-ionic contrast agent, was administered at a rate of $3.5 \mathrm{~mL} / \mathrm{s}$; arterial phase cranial images with slice thickness of $0.625 \mathrm{~mm}, 120 \mathrm{kV}, 400$ $-500 \mathrm{~mA}$, pitch 0.98 and rotation time $0.4 \mathrm{~s}$ were obtained from the skull base level to the vertex at $25 \mathrm{~s}$.

\section{Image interpretation}

Image interpretation was performed using a workstation with OsiriX-64 bit software (Lite Digital Imaging and Communications in Medicine Viewer, version 5.6, Geneva, Switzerland). Images were examined independently by 2 radiologists. An interventional radiologist (A.I.S.) with 17 years of experience and a senior radiology resident (T.B., $5^{\text {th }}$ year radiology resident) performed image interpretation. Multiplanar reformat series were reconstructed for each dataset. Axial, coronal, and sagittal reformatted images were interpreted as maximum intensity projections with $10 \mathrm{~mm}$ slice thickness and volume rendering techniques.

\section{The definitions and measuraments of morphological parameters}

Morphological measurements of the ICA supraclinoid segment, MCA M1 segment, dominant truncus and recessive truncus were taken. Aneurysmal bifurcation and non-aneurysmal contralateral MCA bifurcation were compared in terms of proximal and distal artery diameters, rates of these diameters, and bifurcation angles.

The MCA was segmented into three parts, M1 (originating from the terminal bifurcation of the ICA and terminating at the MCA bifurcation), dominant and recessive truncus (originating at the MCA bifurcation and terminating cerebral cortex). The larger trunk was considered the dominant trunk. ICA supraclinoid segment, MCA M1 segment, dominant truncus and recessive truncus diameters were measured on multiplanar reconstruction images. ICA supraclinoid segment diameter was measured $5 \mathrm{~mm}$ proximal to the ICA apex, MCA M1 segment diameter was measured $5 \mathrm{~mm}$ proximal to the bifurcation apex; superior and inferior truncus diameters were measured $5 \mathrm{~mm}$ proximal to the bifurcation apex. After adjusting the planes parallel to the axis of the artery in the sagittal 
and coronal planes, the diameter was measured in the axial plane. Where the shape of the vessel appears closest to the circle in a plane perpendicular to the diameter flow axis, it was averaged by measuring from 3 or 4 different axes.

In this study, the ratio of M1 diameter to the sum of the dominant and recessive truncus diameters was defined as DA, the ratio of upper truncus diameter to lower truncus diameter as $K A$, the ratio of dominant truncus diameter to $\mathrm{M} 1$ segment diameter as $\mathrm{BA}$, the ratio of recessive truncus diameter to $M 1$ segment diameter as CA and the ratio of M1 diameter to ICA diameter as LA.

The angle between the MCA M1 segment and the dominant truncus was called $\Phi 1$ and the angle between the MCA M1 segment and the recessive truncus was $\Phi 2$. The sum of the angles $\Phi 1$ and $\Phi 2$ was defined as the bifurcation angle. In each patient, the M1 segment plane continued laterally on the sagittal planes. The angle between this plane and the corresponding truncus plane was measured by angle measurement tool of the software. $\Phi 1, \Phi 2$ and bifurcation angle were recorded.

Interobserver reproducibility was evaluated in 15 randomly selected subjects. The mean interobserver difference was $0.06 \pm 0.39 \%$ (95\% limits of agreement), the mean intraobserver difference was $0.05 \pm 0.32 \%$ (95\% limits of agreement).

\section{Statistical analysis}

Statistical Package for Social Sciences for Windows, version 22 (SPSS Inc., Chicago, IL, USA) was used for statistical analysis. For the analysis of bifurcations, it was divided into two categories as the aneurysmal side and the contralateral side without aneurysm. Continuous variables with normal distribution were reported as mean \pm standard deviation and continuous data with abnormal distribution were reported as median (minimum-maximum). Categorical variables were reported as frequency (\%).

Pearson $\chi^{2}$ test or Fisher's exact test were used to compare categorical variables. In the comparison of continuous variables, Student $t$ test was used for continuous normally distributed data and Wilcoxon rank sum test was used for non-normally distributed data. They were tested independently.

In the statistical analysis of the study, $p$ value $<0.05$ was considered statistically significant. Receiver operating characteristic (ROC) curves were used to determine the optimal cut-off values of predictor.
Table 1. Bilateral comparison of artery diameter and diameter ratios associated with aneurysm

\begin{tabular}{lccc}
\hline & $\begin{array}{c}\text { Aneurysm } \\
\text { side }\end{array}$ & $\begin{array}{c}\text { Contralateral } \\
\text { side }\end{array}$ & $\mathbf{P}$ \\
\hline M1 segment diameter [mm] & $2.2 \pm 0.52$ & $2.2 \pm 0.52$ & 0.82 \\
$\begin{array}{lccc}\text { Dominant truncus diameter } \\
\text { [mm] }\end{array}$ & $1.82 \pm 0.45$ & $1.81 \pm 0.48$ & 0.94 \\
Recessive truncus diameter & $1.23 \pm 0.40$ & $1.25 \pm 0.41$ & 0.82 \\
[mm] & & & \\
ICA diameter [mm] & $3.1 \pm 0.64$ & $3 \pm 0.6$ & 0.22 \\
LA ratio & $0.71 \pm 0.14$ & $0.74 \pm 0.14$ & 0.19 \\
KA ratio & $0.68 \pm 0.17$ & $0.70 \pm 0.19$ & 0.83 \\
DA ratio & $0.74 \pm 0.14$ & $0.74 \pm 0.12$ & 0.86 \\
BA ratio & $0.83 \pm 0.18$ & $0.82 \pm 0.15$ & 0.97 \\
CA ratio & $0.55 \pm 0.14$ & $0.56 \pm 0.15$ & 0.95 \\
\hline
\end{tabular}

$\mathrm{DA}$ ratio - the ratio of $\mathrm{M} 1$ diameter to the sum of the dominant and recessive truncus diameters; KA ratio - the ratio of upper truncus diameter to lower truncus diameter; $\mathrm{BA}$ ratio - the ratio of dominant truncus diameter to $\mathrm{M} 1$ segment diameter; $\mathrm{CA}$ - the ratio of recessive truncus diameter to $\mathrm{M} 1$ segment diameter; $\mathrm{LA}$ - the ratio of $\mathrm{M} 1$ diameter to ICA diameter as LA

\section{RESULTS}

The study group consisted of 74 patients $(62.2 \%$ female) aged between 24 and 79 years (mean age 58.41 years). $52.7 \%$ (39) of the aneurysms were on the right, while $47.3 \%$ (35) were localised to the left. $66.2 \%$ (49) of the aneurysms were not ruptured whereas $33.8 \%$ (25) were ruptured.

There was no statistically significant difference between ICA, MCA M1 segment, dominant and recessive truncus diameters and the ratios of these diameters in the aneurysmal side and in the contralateral bifurcation side as control group (Table 1).

The dominant truncus angle ( $\Phi 1$ ) on the aneurysm side was $81.5 \pm 33.1^{\circ}$, whereas this angle $(\Phi 1)$ on the contralateral side was $55.8 \pm 21.1^{\circ}(p<0.0001)$. The recessive truncus angle (\$2) on the aneurysm side was $98.2 \pm 30.9^{\circ}$, while this angle ( $(2)$ on the contralateral side was $68.2 \pm 25.7^{\circ}(p<0.0001)$. The bifurcation angle on the aneurysm side was $179.7 \pm$ $\pm 42.9^{\circ}$, as it was $124 \pm 33.3^{\circ}$ on the contralateral side $(p<0.0001)$. When the dominant, recessive truncus and bifurcation angles were compared to the aneurysm side and the contralateral side, it was found that the mean of all three angles on the aneurysm side was significantly higher $(p<0.0001)$ (Table 2$)$.

The diagnostic feature of bifurcation angle for predicting aneurysm formation was examined by ROC curve analysis (Fig. 1). Significant limit value was determined. Then sensitivity, specificity, positive predictive value and negative predictive value were 
Table 2. Middle cerebral artery (MCA) bifurcation angle measurements on ipsilateral and contralateral sides

\begin{tabular}{lccc}
\hline & $\begin{array}{c}\text { Aneurysmal } \\
\text { MCA }(\mathbf{n}=74)\end{array}$ & $\begin{array}{c}\text { Contralateral } \\
\text { MCA }(\mathbf{n}=74)\end{array}$ & P \\
\hline$\Phi 1$ & $81.5 \pm 33.1$ & $55.8 \pm 21.1$ & $<0.0001$ \\
$\Phi 2$ & $98.2 \pm 30.9$ & $68.2 \pm 25.7$ & $<0.0001$ \\
Bifurcation angle & $179.7 \pm 42.9$ & $124.18 \pm 33.37$ & $<0.0001$ \\
\hline
\end{tabular}

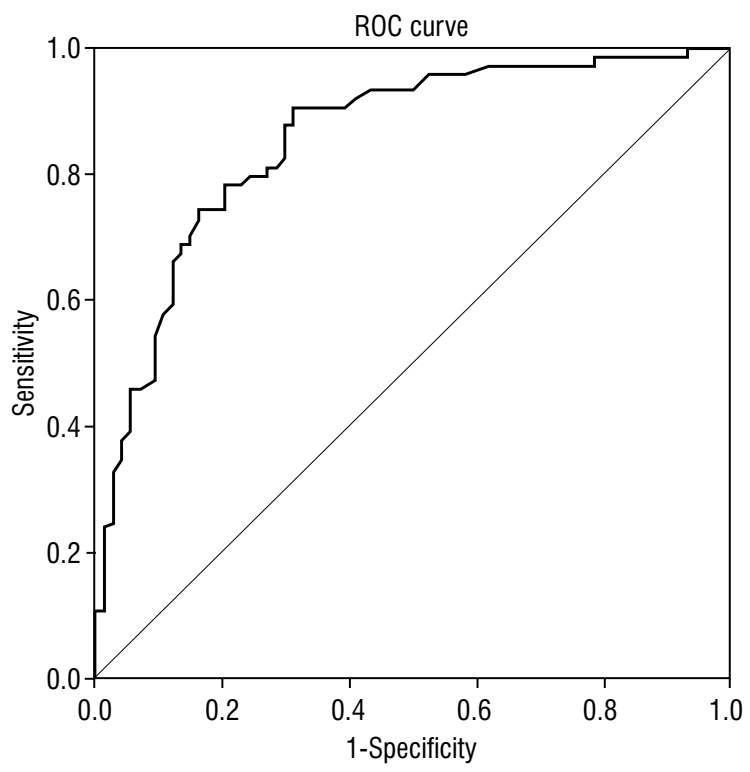

Diagonal segments are produced by ties

Figure 1. Diagnostic feature of the sum of upper and lower angle measurement for predicting aneurysm formation according to receiver operating characteristic (ROC) curve analysis.

Table 3. Diagnostic feature of bifurcation angle for predicting aneurysm formation, values of three angles obtained according to receiver operating characteristic curve analysis

\begin{tabular}{ccccc}
\hline $\begin{array}{l}\text { Limit } \\
\text { value }\end{array}$ & Sensitivity & Specificity & $\begin{array}{c}\text { Positive pre- } \\
\text { dictive value }\end{array}$ & $\begin{array}{c}\text { Negative pre- } \\
\text { dictive value }\end{array}$ \\
\hline 146.5 & $78.4 \%$ & $78.4 \%$ & $78.4 \%$ & $78.4 \%$ \\
147.5 & $78.4 \%$ & $79.7 \%$ & $79.5 \%$ & $78.7 \%$ \\
148.5 & $77 \%$ & $79.7 \%$ & $79.2 \%$ & $77.6 \%$ \\
\hline
\end{tabular}

calculated for this value. In the evaluation of the area under the curve (AUC), when the type 1 error level was below $5 \%$, the diagnostic value of the test was interpreted as statistically significant. In the ROC analysis, when the bifurcation angle of $147.5^{\circ}$ was accepted as the limit value, $78.4 \%$ sensitivity, $79.7 \%$ specificity, $79.5 \%$ positive predictive value and $78.7 \%$ negative predictive value were determined (AUC: 0.85 ; Table 3).

\section{DISCUSSION}

The pathophysiological mechanism of IA formation is controversial. The development of IAs is associated with acquired factors such as smoking and hypertension, as well as with congenital and genetic factors [23]. If the patient has certain risk factors (female gender, smoking, alcohol, age and hypertension) or family history, aneurysm development is more likely [14]. However, it is almost impossible to predict precisely the localisation of the aneurysm, and thus the onset and early development of the aneurysm. In addition to the genetic and acquired factors mentioned above, haemodynamic stress is thought to play an important role in the formation of aneurysms by triggering focal degenerative mechanisms in the vessel wall [17]. Middle cerebral artery bifurcation has a very complex morphology due to angulations as well as varying diameters and variations of M1 segment and truncal branches. Because of this complex morphology, haemodynamic stress in different localizations of this structure is not homogeneous [18]. In order to minimise the confounding effects of acquired risk factors in our study, we compared the bifurcation in the aneurysmal side and contralateral side without aneurysm in the same patient. We excluded patients with bilateral aneurysms.

It is essential that the branching and bifurcation zones in the cerebral arteries are optimally arranged to generate a constant wall shear stress (WSS) by consuming minimum energy along the main artery and branches. Haemodynamic factors such as WSS are affected by the geometry of the vascular tree [3]. The optimal principle of minimum work minimises wall tension stress due to both vascular diameters and bifurcation angles [11]. Ingebrigtsen et al. [7] assumed that normal MCA bifurcations would follow the minimum work principles and the presence of an aneurysm would be associated with deviations from the optimum bifurcation geometry.

The bifurcation apex is the maximum stress zone in the artery due to the direct effect of blood flow. This region is exposed to high WSS variations that are believed to cause endothelial damage to the vessel wall. As the angle of bifurcation increases, the forces applied to the lateral branches try to balance each other more but compensate less for the forces applied to the apex of the parent artery [15]. It has been shown that high pressure caused by increased bifurcation angle in apex may be associated with endothelial dysfunction and aneurysm progression 
secondary to endothelial proliferation and apoptosis [8]. In their study, Roach et al. [15] found that aneurysms localised to wide bifurcation angles were associated with a large stagnation area and high WSS at the bifurcation apex. These haemodynamic changes could cause them to grow more than those settled in narrow-angle bifurcations [15]. Finlay et al. [4] described a collagen tendon-like medial pad that is thought to protect the bifurcation apex where flow is divided into side branches and has the highest WSS and spatial wall shear stress gradient (WSSG) [4]. Meng et al. [10] reported the presence of an "intimal pad" in the stroke area of the flow jet in the bifurcation. In both studies, it was found that as the bifurcation angles increased, the stroke area of the blood moved away from the bifurcation apex where the arterial wall was preserved, and the blood flow forming larger vortices needed a longer distance to return to the laminar state. This has been reported to cause greater damage to the vessel wall adjacent to the dense collagen fibre area. It has been reported that these dynamics changes in wide bifurcation angles lead to aneurysm formation as a result of high WSS and WSSG exposure in the vulnerable artery wall around the bifurcation apex [4].

The effects of MCA M1 segment, branch diameters and branch angles can be conceptualised as haemodynamics of the bifurcation point in the artery. Since the bifurcation angles are relatively larger in aneurysmatic bifurcations, we assume that blood flow has to make a deeper deviation at this point. In studies evaluating bifurcation geometry in Willis polygon, aneurysmal bifurcations have been reported to have wider bifurcation angles than non-aneurysmal bifurcations [2, 7]. Sadatomo et al. [16] reported that aneurysmal MCA bifurcations had narrower lateral angles than non-aneurysmal bifurcations in their studies evaluating MCA bifurcations. In this study, unlike other studies, the lateral angle which is accepted as the angle associated with the aneurysm, is complementary to the angle in other studies. Therefore, the correlation with the narrow angle was reported as the opposite of our study. In our study, truncal angles and bifurcation angles on the side of the aneurysmal MCA bifurcation were significantly wider than the control group $(p<0.0001)$.

Baharoğlu et al. [1] reported that changes in bifurcation vessel geometry were associated with increased risk of aneurysm formation by altering haemodynamic forces at the apex of the bifurcation in their study of localised aneurysms in MCA bifurcation. In this study, $140^{\circ}$ for bifurcation angle (93\% sensitivity and $93 \%$ specificity, AUC: 0.98$), 69^{\circ}$ for upper trunk angle ( $63 \%$ sensitivity and $96 \%$ specificity, AUC: 0.84$)$ and $83^{\circ}$ for lower trunk angle ( $78 \%$ sensitivity and $91 \%$ specificity, AUC: 0.91 ) have been reported [1]. Total bifurcation angle has been reported to perform best in differentiating aneurysmal and non-aneurysmal MCA. In another study, Gao et al. [5] reported that stent-mediated treatment effectively reduced the angle of bifurcation in the postoperative period and reduced wall damage caused by abnormal hemodynamic stress at the bifurcation apex. Therefore, not only the individual angles between the parent artery and the branches ( $\Phi 1$ and $\Phi 2)$, but also the bifurcation angle were examined. In our study, when the $147.5^{\circ}$ bifurcation angle was accepted as the limit value, $78.4 \%$ sensitivity, $79.7 \%$ specificity, $79.5 \%$ positive predictive value and $78.7 \%$ negative predictive value were determined (AUC: 0.85 ). The data of our study is consistent with the results of the study of Baharoğlu et al. [1].

Our other hypothesis is that if the sum of the dominant and recessive truncus diameters forming the distal bed is larger than the diameter of the M1 segment, the blood can proceed freely without causing a haemodynamic imbalance in the distal bifurcation region, but in the opposite case the changing flow dynamics might trigger the development of the aneurysm. According to the principle of flow protection, the distal bed should be at least equal to the proximal so blood can flow freely without encountering a high resistance [12]. The smaller main vessel diameter causes higher jet flow at the bifurcation apex and increases the haemodynamic stress which the arterial wall is exposed [13]. Therefore, simple morphological parameters like large bifurcation angles, disproportionate diameters and haemodynamic changes, may be useful in predicting aneurysm formation in high-risk patients. In the study examining the relationship between anterior communicating artery ( $A C O A)$ formation and environmental geometry, a positive correlation was observed not only between $\mathrm{A} 1$ and $\mathrm{A} 2$ diameter ratio and aneurysm formation but also the incidence of $A 1$ dominance to feed both A2 [20]. Soylu et al. [19] reported that increased contralateral ICA/A1 ratio, increased ipsilateral A1/A2 ratio and narrow bifurcation angle were the most important determinants for aneurysm development in their study evaluating the morphological factors 
affecting ACoA aneurysms. In this study, we did not find any significant difference when we compared ICA, M1 segment, dominant and recessive truncus diameters and the ratio of these diameters. In our study group, normal physiological flow pattern was present in M1 and its branches. Therefore there was no effect of flow rate in main and branch arteries on the development of aneurysm.

\section{Limitations of the study}

The main limitation of this study is related to retrospective design. We cannot conclude that a larger bifurcation angle causes aneurysm formation. Because of the lack of data on geometry before and after aneurysm formation, we cannot ignore the possibility that aneurysm formation affects adjacent vessel geometry. Therefore, all outcome about the parameters examined can only be related to the presence of aneurysm and are not necessarily predictors of the risk of occurrence. This study, it does not give information about the clinical course of the disease or haemodynamic properties of the flow. Also the measurements are performed manually in our study. Although the results vary slightly, this is a much more applicable technique in the clinical setting. Care is taken to avoid any changes in diameter measurements between observers but the resulting bias cannot be ruled out.

\section{CONCLUSIONS}

In conclusion, our study showed that the presence of MCA aneurysms was significantly associated with large bifurcation angles. In patients with clinical risk factors large bifurcation angle might be interpreted as additional risk factor. Measurement of these simple morphological factors can be easily performed by radiologists.

\section{REFERENCES}

1. Baharoglu MI, Lauric A, Safain MG, et al. Widening and high inclination of the middle cerebral artery bifurcation are associated with presence of aneurysms. Stroke. 2014; 45(9): 2649-2655, doi: 10.1161/STROKEAHA.114.005393, indexed in Pubmed: 25116869.

2. Bor AS, Velthuis BK, Majoie CB, et al. Configuration of intracranial arteries and development of aneurysms: a follow-up study. Neurology. 2008; 70(9): 700-705, doi: 10.1212/01.wnl.0000302176.03551.35, indexed in Pubmed: 18299521.

3. Can A, Ho AL, Dammers R, et al. Morphological parameters associated with middle cerebral artery aneurysms. Neurosurgery. 2015; 76(6): 721-6; discussion 726, doi: 10.1227/ NEU. 0000000000000713 , indexed in Pubmed: 25734323.
4. Finlay HM, Whittaker P, Canham PB. Collagen organization in the branching region of human brain arteries. Stroke. 1998; 29(8): 1595-1601, doi: 10.1161/01.str.29.8.1595, indexed in Pubmed: 9707199.

5. Gao B, Baharoglu MI, Cohen AD, et al. Y-stent coiling of basilar bifurcation aneurysms induces a dynamic angular vascular remodeling with alteration of the apical wall shear stress pattern. Neurosurgery. 2013; 72(4): 617-29; discussion 628, doi: 10.1227/NEU.0b013e3182846d9f, indexed in Pubmed: 23277371.

6. Greenberg M. Handbook of Neurosurgery. 6th ed. New York 2006: 289-365.

7. Ingebrigtsen $\mathrm{T}$, Morgan MK, Faulder $\mathrm{K}$, et al. Bifurcation geometry and the presence of cerebral artery aneurysms. J Neurosurg. 2004; 101(1): 108-113, doi: 10.3171/ jns.2004.101.1.0108, indexed in Pubmed: 15255260.

8. Kondo S, Hashimoto N, Kikuchi $\mathrm{H}$, et al. Apoptosis of medial smooth muscle cells in the development of saccular cerebral aneurysms in rats. Stroke. 1998; 29(1): 181-189, doi: 10.1161/01.str.29.1.181, indexed in Pubmed: 9445349.

9. Longstreth WT, Nelson LM, Koepsell TD, et al. Cigarette smoking, alcohol use, and subarachnoid hemorrhage. Stroke. 1992; 23(9): 1242-1249, doi: 10.1161/01. str.23.9.1242, indexed in Pubmed: 1519278.

10. Meng H, Wang Z, Hoi $Y$, et al. Complex hemodynamics at the apex of an arterial bifurcation induces vascular remodeling resembling cerebral aneurysm initiation. Stroke. 2007; 38(6): 1924-1931, doi: 10.1161/STROKEAHA.106.481234, indexed in Pubmed: 17495215.

11. Murray $C D$. The physiological principle of minimum work applied to the angle of branching of arteries. J Gen Physiol. 1926; 9(6): 835-841, doi: 10.1085/jgp.9.6.835, indexed in Pubmed: 19872299.

12. Murray CD. The physiological principle of minimum work: I. The vascular system and the cost of blood volume. Proc Natl Acad Sci U S A. 1926; 12(3): 207-214, doi: 10.1073/ pnas.12.3.207, indexed in Pubmed: 16576980.

13. Przelomski MM, Fisher M, Davidson Rl, et al. Unruptured intracranial aneurysm and transient focal cerebral ischemia: a follow-up study. Neurology. 1986; 36(4): 584-587, doi: 10.1212/wnl.36.4.584, indexed in Pubmed: 3960339.

14. Raps EC, Rogers JD, Galetta SL, et al. The clinical spectrum of unruptured intracranial aneurysms. Arch Neurol. 1993; 50(3): 265-268, doi: 10.1001/archneur.1993.00540030031010, indexed in Pubmed: 8442705.

15. Roach MR, Scott S, Ferguson GG. The hemodynamic importance of the geometry of bifurcations in the circle of Willis (glass model studies). Stroke. 1972; 3(3): 255-267, doi: 10.1161/01.str.3.3.255, indexed in Pubmed: 5034974.

16. Sadatomo T, Yuki K, Migita K, et al. Differences between middle cerebral artery bifurcations with normal anatomy and those with aneurysms. Neurosurg Rev. 2013; 36(3): 437-445, doi: 10.1007/s10143-013-0450-5, indexed in Pubmed: 23354785.

17. Schievink WI, Schaid DJ, Rogers HM, et al. On the inheritance of intracranial aneurysms. Stroke. 1994; 25(10): 2028-2037, doi: 10.1161/01.str.25.10.2028, indexed in Pubmed: 8091449. 
18. Shojima M, Oshima M, Takagi K, et al. Role of the bloodstream impacting force and the local pressure elevation in the rupture of cerebral aneurysms. Stroke. 2005; 36(9): 1933-1938, doi: 10.1161/01.STR.0000177877.88925.06, indexed in Pubmed: 16081858.

19. Soylu Ai, Ozturk M, Akan H. Can vessel diameters, diameter ratios, and vessel angles predict the development of anterior communicating artery aneurysms: A morphological analysis. J Clin Neurosci. 2019; 68: 250-255, doi: 10.1016/j.jocn.2019.07.024, indexed in Pubmed: 31358430.

20. Tarulli E, Fox AJ. Potent risk factor for aneurysm formation: termination aneurysms of the anterior communicating artery and detection of $\mathrm{A} 1$ vessel asymmetry by flow dilution.
Am J Neuroradiol. 2010; 31(7): 1186-1191, doi: 10.3174/ ajnr.A2065, indexed in Pubmed: 20360345.

21. Taylor CL, Yuan Z, Selman WR, et al. Cerebral arterial aneurysm formation and rupture in 20,767 elderly patients: hypertension and other risk factors. J Neurosurg. 1995; 83(5): 812-819, doi: 10.3171/jns.1995.83.5.0812, indexed in Pubmed: 7472548.

22. Wardlaw JM, White PM. The detection and management of unruptured intracranial aneurysms. Brain. 2000; 123(2): 205-221, doi: 10.1093/brain/123.2.205.

23. Wiebers DO, Whisnant JP, Sundt TM, et al. The significance of unruptured intracranial saccular aneurysms. J Neurosurg. 1987; 66(1): 23-29, doi: 10.3171/ jns.1987.66.1.0023, indexed in Pubmed: 3783255. 